\title{
Morusin suppresses A549 cell migration and induces cell apoptosis by downregulating the expression of COX-2 and VEGF genes
}

\author{
XIAO-LU YIN, YI LV, SHU WANG and YU-QING ZHANG \\ Silk Biotechnology Laboratory, School of Basic Medical and Biological Sciences, \\ Soochow University, Suzhou, Jiangsu 215123, P.R. China
}

Received October 24, 2017; Accepted May 3, 2018

DOI: $10.3892 /$ or.2018.6431

\begin{abstract}
The present study aimed to examine the inhibitory effects of morusin on the human lung cancer cell line A549. Various doses of morusin were applied to A549 cells and the effects were assessed by wound-healing and MTT assays, flow cytometry analysis of apoptosis, a mitochondrial membrane potential assay and RT-PCR. The results indicated that the concentrations of 10 and $30 \mu \mathrm{g} / \mathrm{ml}$ morusin significantly inhibited A549 cells and signs of apoptosis were observed. In addition, the wound-healing assay results revealed that morusin inhibited cell migration. Flow cytometry analysis demonstrated that the rates of apoptosis were 16.46, 55.80 and $70.80 \%$ following treatment with 1,10 and $30 \mu \mathrm{g} / \mathrm{ml}$ of morusin, respectively, and that the mitochondrial membrane potentials also decreased with the increase of morusin. Furthermore, morusin increased the antioxidant activities of the A549 cells. RT-PCR analysis revealed that the expression levels of COX-2 and VEGF were downregulated following morusin treatment. In conclusion, morusin significantly inhibited the proliferation of the lung cancer cell line A549, and may have affected the invasion and migration of the cells by downregulating the expression of tumor angiogenesis-related genes.
\end{abstract}

\section{Introduction}

Lung cancer is a highly prevalent primary pulmonary malignancy that originates in the bronchial mucosa and has a very high mortality rate (1). Non-small cell lung cancer accounts for $\sim 80 \%$ of all lung cancer cases (2). To date the primary treatment of intermediate- and advanced-stage lung cancer is chemotherapy or radiotherapy. However, the effects of these treatments are limited with a low survival rate and serious

Correspondence to: Professor Yu-Qing Zhang, Silk Biotechnology Laboratory, School of Basic Medical and Biological Sciences, Soochow University, No. 199, 702-2303 Room, Renai Road, Dushuhu Higher Education Town, Suzhou, Jiangsu 215123, P.R. China

E-mail: sericult@suda.edu.cn

Key words: morusin, A549, cell apoptosis, mitochondrial membrane potential, tumor angiogenesis-related genes side-effects often accompany such treatments (3). Thus, the identification of new agents that produce minimal side-effects and have a good treatment efficacy is a potential mean to improve the effects of therapy and overall patient survival.

It is well known that different parts of mulberry (Morus) trees have pharmaceutical use. The root bark (cortex mori) is widely used in traditional Chinese medicine. Cortex mori contains a variety of chemical components, including flavonoids (4), alkaloids (5), coumarin (6) and stilbenes (7-9). Morusin is a flavone that was first isolated from the root bark of Morus alba L. (10). Flavones exhibit many kinds of medical functions, such as antitumor and antioxidant activities. Research has revealed that morusin has anti-leukemic $(11,12)$, anti-bacterial (13), anti-convulsive (14), anti-HIV (15) and anti-Alzheimer's $(16,17)$ functions, though the main part of the effect of morusin lies in its antitumor activity. Early in 1989, Yoshizawa et al reported the inhibitory effect of morusin on tumor promotion by teleocidin. These results indicated that morusin inhibited the specific binding of $\left(\left[{ }^{3} \mathrm{H}\right] \mathrm{TPA}\right.$ (12-O-tetradecanoyl-phorbol-13-acetate) to a particulate fraction of mouse skin and inhibited teleocidin-induced activation of protein kinase $\mathrm{C}$. Thus, morusin is a novel antitumor agent (18). Fujiki et al reported that morusin inhibited tumor promotion in two-stage mouse skin carcinogenicity tests and may be used for the future chemoprevention of cancer (19). A recent study indicated that morusin inhibited cell proliferation and tumor growth by downregulating c-Myc in human gastric cancer (20). Furthermore, other studies have illustrated that morusin exerted a strong inhibitory effect on the growth of HT-29 rectosigmoid adenocarcinoma cells via the activation of caspases and the mitochondrial pathway of cell apoptosis $(21,22)$. Additionally, morusin suppressed the signal transducer and activator of transcription 3 (STAT3) and nuclear factor- $\kappa \mathrm{B}(\mathrm{NF}-\kappa \mathrm{B})$ signaling pathways, which modulate the expression of proteins involved in the invasion process in human SK-Hep1 hepatocellular carcinoma cells (23). In addition, blockage of the STAT3 signaling pathway by morusin induced apoptosis and inhibited invasion in human pancreatic tumor cells (AsPC-1, BxPC-3, MIA PaCa-2, and PANC-1) (24). Morusin can also exert inhibitory effects on stem cell growth and migration in human cervical cancer (25). Furthermore, our group has investigated the effects of morusin on the growth and pathological changes of $\mathrm{H} 22$ hepatocarcinoma cells transplanted into a mouse model. This result indicated 
that morusin upregulated the expression of caspase- 3 and suppressed NF- $\mathrm{B}$ signaling (26). Our group also observed that morusin downregulated the expression of $\mathrm{NF}-\kappa \mathrm{B}$, upregulated caspase-3, caspase-9, p-ERK1/2 and p-JNK as well as induced the apoptosis of human hepatoma Bel-7402 cells via the mitochondrial and MAPK pathways (27). Although A549 cells have been researched extensively, the study of the tumor angiogenesis-related genes COX-2 and VEGF has been limited. In the present study, we examined the inhibitory effects of morusin on the growth and migration of human A549 lung cancer cells. Our results indicated that morusin induced mitochondrial membrane permeability changes and downregulated angiogenesis-related genes in the A549 cells. The present study indicated that morusin was a potential drug candidate for the chemotherapy of lung cancer.

\section{Materials and methods}

Materials. Morusin (2-[2,4-dihydroxyphenyl]-5-hyd roxy-8, 8-dimethyl-3-[3-methyl-1-2-butenyl]-4H,8Hbenzo[1,2-b; 3,4-b']dipyran-4-one; $\mathrm{C}_{25} \mathrm{H}_{24} \mathrm{O}_{6}$; purity $\geq 98 \%$ ) was purchased from Chengdu Ruifensi Biotechnology Co., Ltd. (Chengdu, China). Morusin was dissolved in DMSO and diluted in water. A final concentration of $<0.1 \%$ DMSO was used in the experiments.

Cell line. The human lung cancer cell line A549 was purchased from Nanjing KeyGen BioTech Co., Ltd. (Nanjing, China). The A549 cells were cultured in RPMI-1640 medium supplemented with $10 \%$ fetal bovine serum (FBS; Invitrogen; Thermo Fisher Scientific, Inc., Waltham, MA, USA), 100 IU/ml penicillin and $100 \mathrm{IU} / \mathrm{ml}$ streptomycin (Beyotime Institute of Biotechnology, Haimen, China). The cells were maintained at $37^{\circ} \mathrm{C}$ in a humidified atmosphere of $5 \% \mathrm{CO}_{2}$.

MTT assay. MTT colorimetric assays were performed to evaluate the cytotoxic effect of morusin on the A549 cells (28). Firstly, the cells were seeded in 96-well plates at a $1 \times 10^{5}$ cells/well; the control group was treated with $\mathrm{DMSO}+\mathrm{PBS}$, and the other groups were treated with morusin at 1,10 and $30 \mu \mathrm{g} / \mathrm{ml}$ concentrations for $48 \mathrm{~h}$ at $37^{\circ} \mathrm{C}$. Following the exposure period, the cells were incubated with $20 \mu \mathrm{l}$ MTT $(0.5 \mathrm{mg} / \mathrm{ml})$ for an additional $4 \mathrm{~h}$. The formazan crystals were dissolved in $150 \mu 1$ DMSO. The absorbance was assessed with a spectrophotometer (SpectraMax M5; Molecular Devices, San Jose, CA, USA) at $495 \mathrm{~nm}$. Secondly, the cells were seeded in 96-well plates at a specific density and treated with morusin at 1,10 and $30 \mu \mathrm{g} / \mathrm{ml}$ concentrations at $37^{\circ} \mathrm{C}$ for 24,48 and $72 \mathrm{~h}$, and the remaining steps were performed as above-described. The inhibition ratio was calculated as follows: $\left(\mathrm{A}_{\text {con }}-\mathrm{A}_{\text {exp }}\right) / \mathrm{A}_{\text {con }} \times 100 \%$, where $\mathrm{A}_{\exp }$ and $\mathrm{A}_{\text {con }}$ are the absorbance values of the treated and untreated cells, respectively.

Wound-healing assay. A wound-healing assay was used to assess cell migration. The A549 cells were seeded into 6 -well plates and incubated for $24 \mathrm{~h}$. A 'wound' was made by manually scraping the monolayer in the middle of the each well with a $10-\mu 1$ pipette tip. Cells were added with fresh morusin-containing medium or medium along (control), and
Table I. The primer sequences of the genes used in RT-PCR.

\begin{tabular}{ll}
\hline Gene & \multicolumn{1}{c}{ Primer sequences (5' to $3 ')$} \\
\hline COX-2 & $\begin{array}{l}\text { Forward: AGCATCTACGGTTTGCTGTG } \\
\text { Reverse: CCTGTTTAAGCACATCGCAT }\end{array}$ \\
VEGF & $\begin{array}{l}\text { Forward: GCAGCTTGAGTTAAACGAACG } \\
\text { Reverse: GGTTCCCGAAACCCTGAG }\end{array}$
\end{tabular}

F, forward; R, reverse.

the cells were incubated for another $24 \mathrm{~h}$. After incubation, the cells were imaged via inverted microscopy (AMG, Mill Creek, WA, USA) to determine the wound-healing rate.

Annexin V-FITC/PI double staining. A549 cells were treated with morusin for $24 \mathrm{~h}$, and then examined via flow cytometry using a commercial Annexin V-FITC/PI Apoptosis Detection kit (Nanjing KeyGen BioTech). Following treatment, the cells were collected and washed twice in PBS, then resuspended in Annexin-binding buffer. The samples were subsequently stained with Annexin V-fluorescein isothiocyanate (FITC) and propidium iodide (PI), and then incubated for $15 \mathrm{~min}$ in the dark. The cells were analyzed by flow cytometry (Becton-Dickinson, San Jose, CA, USA).

Mitochondrial membrane potential $(\Delta \psi)$ assay. $\Delta \psi$ was evaluated using the probe JC-1, which reversibly changes color from green, at $535 \mathrm{~nm}$, to orange, at $590 \mathrm{~nm}$, as $\Delta \psi$ increases. JC-1 has both a monomer and polymer state. It exists in monomer and green fluorescent can be detected in the form of FL-1 channel with FITC when the cells are in high concentration. It exists in polymer and red fluorescent can be detected in the form of FL-2 channel with PE. In brief, the cells were harvested, washed, resuspended in PBS, and stained with JC-1 for $15-20 \mathrm{~min}$ at $37^{\circ} \mathrm{C}$ in the dark. The resuspended solutions were then analyzed by flow cytometry.

Determination of antioxidant activities. The cells were treated with or without different concentrations of morusin for $24 \mathrm{~h}$. The SOD, GSH and T-AOC concentrations were detected with the appropriate T-SOD, GSH-PX and T-AOC commercial kits (Jiancheng Institute of Biotechnology, Co., Ltd, Nanjing, China), according to the manufacturer's instructions.

Real-time PCR analysis. Total RNA was extracted from the cells using TRIzol ${ }^{\circledR}$ reagent (Life Technologies; Thermo Fisher Scientific, Inc.), according to the manufacturer's instructions. The concentration of total RNA was quantified by the absorbance at $260 \mathrm{~nm}$ using a NanoDrop spectrophotometer (Thermo Fisher Scientific, Inc.). Reverse transcription was performed with Prime Script ${ }^{\mathrm{TM}}$ RT reagent kit (Takara Bio, Inc., Otsu, Japan) and the cDNA was prepared for real-time PCR. Real-time PCR was performed in triplicate with SYBR Premix Ex Taq $^{\mathrm{TM}}$ II RT-PCR kit (Takara Bio) using ABI 7900HT Fast Real-Time PCR system (Applied Biosystems Inc., Foster City, CA, USA). The RT-PCR amplification conditions were as follows: $95^{\circ} \mathrm{C}$ for $5 \mathrm{~min} ; 40$ cycles at $95^{\circ} \mathrm{C}$ for 


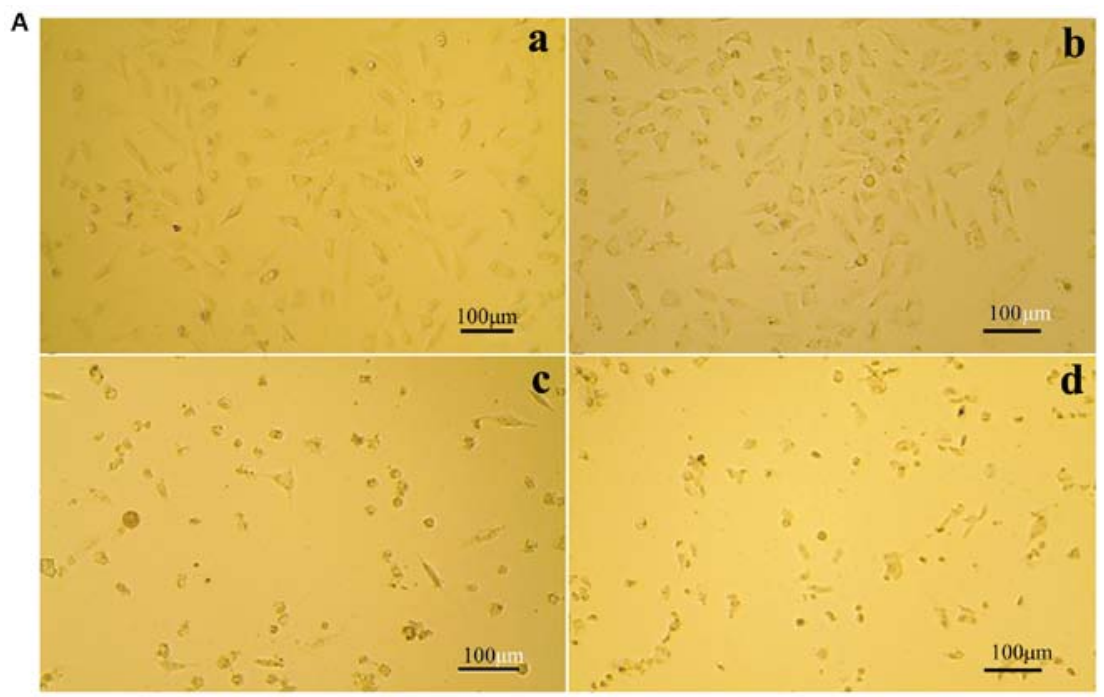

B
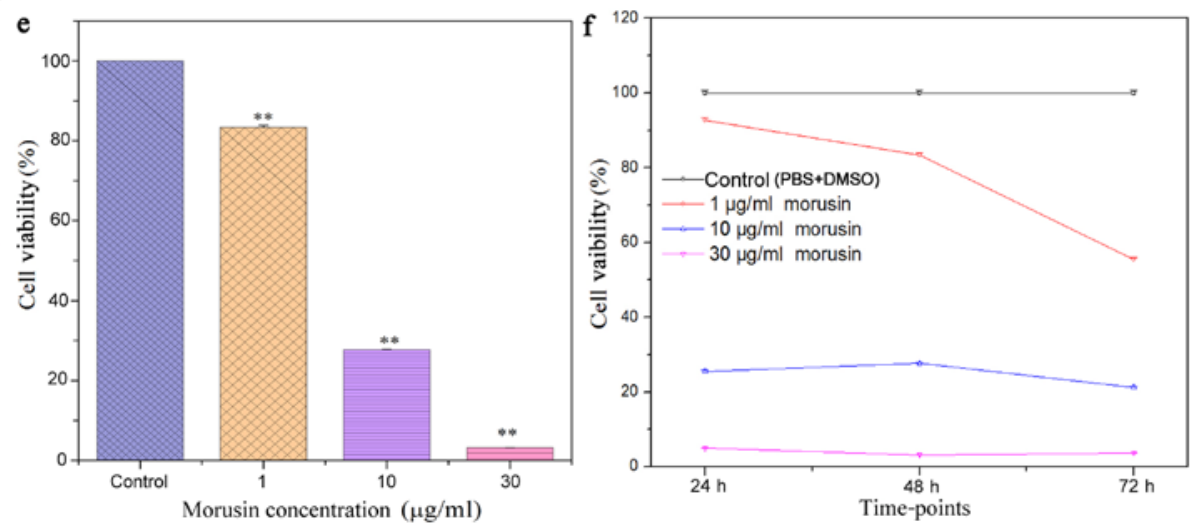

Figure 1. Morphological characteristics of the A549 cells. (A-a) The control group. (A-b) Morusin ( $1 \mu \mathrm{g} / \mathrm{ml})$-treated group. (A-c) Morusin (10 $\mu \mathrm{g} / \mathrm{ml})$-treated group. (A-d) Morusin (30 $\mu \mathrm{g} / \mathrm{ml})$-treated group. The images were obtained at a magnification of x200. (B-e) A 48-h morusin treatment concentration-dependently inhibited the growth of A549 cells. (B-f) Morusin time-dependently inhibited the growth of A549 cells. ${ }^{*} \mathrm{P}<0.05$ and ${ }^{* *} \mathrm{P}<0.01$ vs. the control group.

$15 \mathrm{sec}, 60^{\circ} \mathrm{C}$ for $20 \mathrm{sec}$ and $72^{\circ} \mathrm{C}$ for $40 \mathrm{sec}$. The sequences of the primers are listed in Table I.

Statistical analysis. Data are presented as the mean \pm standard deviation (SD). Statistical significance was tested using one-way ANOVA with Origin 7.5 software (OriginLab, Northampton, MA, USA). $\mathrm{P}<0.05$ was considered to indicate a statistically significant difference.

\section{Results}

Morusin inhibits the growth of human lung cancer cells. The ability of morusin to inhibit the growth of the human A549 lung cancer cell line was evaluated. Following treatment with the various concentrations of morusin $(1,10$ and $30 \mu \mathrm{g} / \mathrm{ml})$ for $24 \mathrm{~h}$, the A549 cells were observed by inverted microscopy (Fig. 1A). The cells of the control group (Fig. 1A-a) were adherent, shiny and exhibited clear polygon forms. However, after treatment with 10 or $30 \mu \mathrm{g} / \mathrm{ml}$ morusin (Fig. 1A-b, c and d), morphological changes were observed, including cell shrinkage and cavitation, and the numbers of cells were significantly decreased. Furthermore, the majority of the cells were floating, and the number of adherent cells had decreased.
The MTT assay was used to determine the cytotoxicity of morusin against the A549 human lung cancer cells. The results are displayed in Fig. 1B-e. Morusin inhibited the growth of A549 cells in a dose-dependent manner. All the treatment groups exhibited significant differences $(\mathrm{P}<0.01)$ compared with the control group. After treatment with $30 \mu \mathrm{g} / \mathrm{ml}$ morusin, the viability of the A549 cells was only $3.21 \%$, and the inhibition rate had reached $96.79 \%$. The results revealed that, when the morusin concentrations reached $30 \mu \mathrm{g} / \mathrm{ml}$, the inhibition rate did not decrease with time. This finding indicated that morusin played an important role in the inhibition of A549 cells. As demonstrated in Fig. 1B-f, the viability of the A549 cells was evaluated after treatment with different concentrations of morusin for 24,48 and $72 \mathrm{~h}$, and when the concentration of morusin was $30 \mu \mathrm{g} / \mathrm{ml}$, cell viability did not reduce with time, indicating that the morusin treatment had reached its maximum effect. At concentrations below $30 \mu \mathrm{g} / \mathrm{ml}$, and with increasing duration, morusin markedly decreased the cell survival rates and increased the inhibition rates of the A549 cells.

Morusin inhibits the migration of A549 cells. As displayed in Fig. 2, A549 cells exhibited wound closure activity, but 


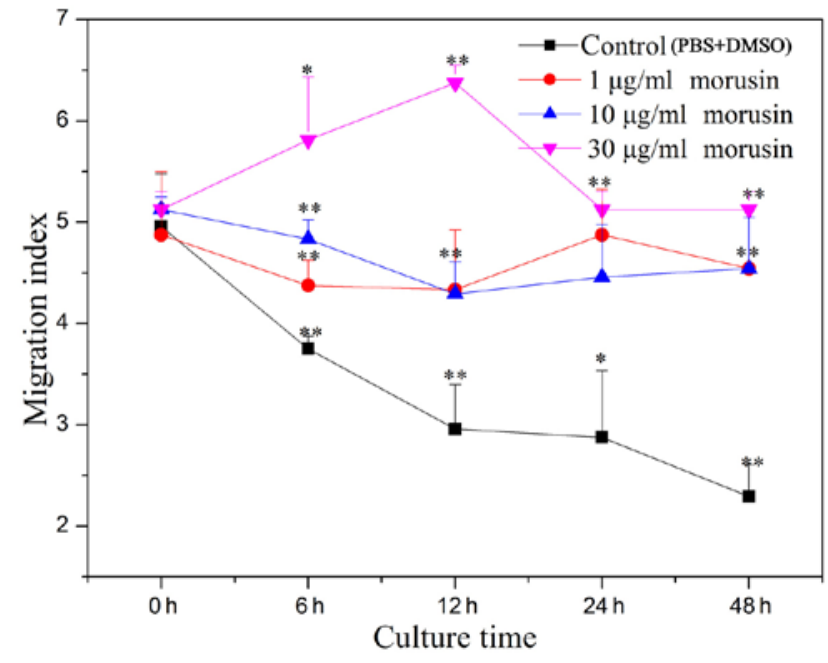

Figure 2. The line chart displays the migration area of the A549 cells treated with morusin compared with the control. ${ }^{*} \mathrm{P}<0.05$ and ${ }^{* * *} \mathrm{P}<0.01$ vs. the control group.

this activity was significantly reduced in the morusin-treated groups in a dose-dependent manner. The degree of wound healing in the untreated group was greater than that in each of the morusin-treated groups. These results indicated that morusin treatment significantly inhibited the migration rates of A549 cells.

Effects of morusin on the apoptosis and $\Delta \psi$ of A549 cells. Annexin V/PI staining was performed to assess morusin-induced apoptosis. As displayed in Fig. 3, significant increases in the ratio of apoptotic cells were observed in the cells treated with different concentrations of morusin $(\mathrm{P}<0.01)$, compared with that of the morusin-untreated cells. The apoptotic rate of the control group was $7.08 \%$, whereas cells treated with $30 \mu \mathrm{g} / \mathrm{ml}$ morusin exhibited an apoptotic rate of $70.80 \%$. The changes in the apoptotic rates in morusin-treated cells were dose-dependent.
The mitochondrion is a key organelle in the process of apoptosis. The loss of $\Delta \psi$ is putatively the initial event that leads to apoptosis (29). To elucidate the association between $\Delta \psi$ and morusin-induced apoptosis, we examined the loss of $\Delta \psi$ by flow cytometry. As illustrated in Fig. $4, \Delta \psi$ was high and JC-1 was mostly in the form of polymers in the control group. Cells were treated with different concentrations of morusin for $24 \mathrm{~h}$, and significant decreases in $\Delta \psi$ and increases of green fluorescence were observed in the A549 cells, in comparison with the untreated cells. Collectively, these results revealed that morusin induced the loss of $\Delta \psi$, which caused changes in the permeability of the mitochondria and eventually induced cell apoptosis.

Morusin increases the antioxidant activities of A549 cells. The levels of antioxidant activity in morusin-treated A549 cells were examined in the present study. As shown in Fig. 5, the antioxidant enzyme levels were increased following treatment with different concentrations of morusin. The results showed that morusin clearly and significantly increased the activity levels of SOD, GSH-PX and T-AOC, in comparison with those of the control group $(\mathrm{P}<0.01)$, in a dose-dependent manner, and thus increased the antioxidant abilities of the A549 cells.

Morusin reduces the expression of the VEGF gene and COX-2 gene in A549 cells. VEGF is a key regulator of vascular permeability and promotes endothelial cell proliferation and migration $(30,31)$. The RT-PCR results revealed that the expression of VEGF was reduced by 18,38 and $56 \%$ following treatment of A549 cells with 1,10 and $30 \mu \mathrm{g} / \mathrm{ml}$ morusin, respectively (Fig. 6A). This indicated that morusin suppressed expression of the VEGF gene, which is related to angiogenesis, in a dose-dependent manner. As displayed in Fig. 6B, the expression of the COX-2 gene was high in the control group. After the cells were treated with different concentrations of morusin, the expression of the COX-2 gene was decreased in a dose-dependent manner. In summary, these results indicated that morusin inhibited the expression of VEGF and COX-2 genes, and thus affected tumor angiogenesis in A549 cells.
A

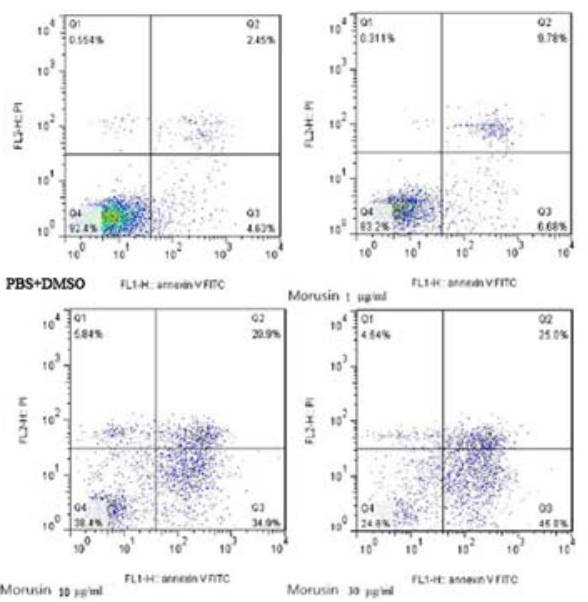

B

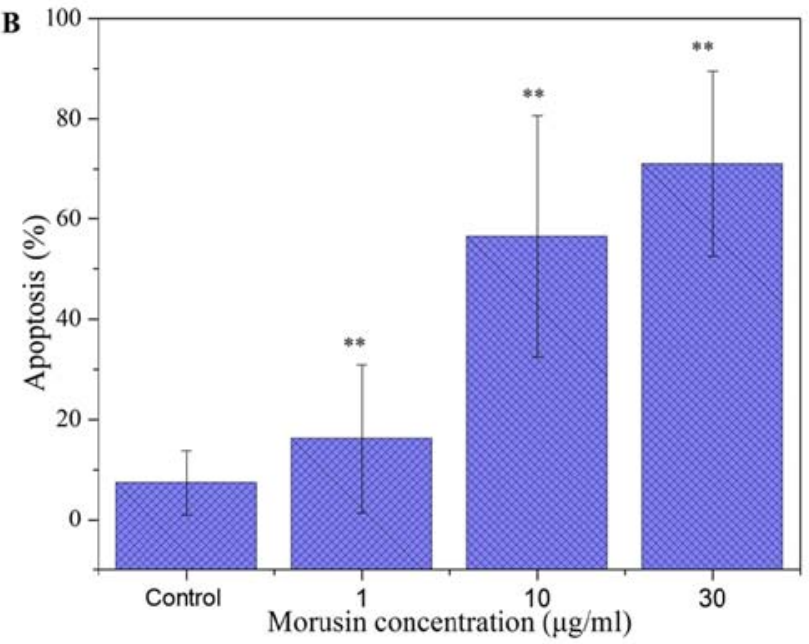

Figure 3. (A) Apoptosis of A549 cells was determined by Annexin V-FITC/PI staining via flow cytometry. The y-axis indicates the PI-labeled population, and the $\mathrm{x}$-axis indicates the FITC-labeled Annexin V-positive cells. (B) Effect of morusin on apoptosis in A549 cells. ${ }^{*} \mathrm{P}<0.05$ and ${ }^{* * *} \mathrm{P}<0.01$ vs. the control group. 
A

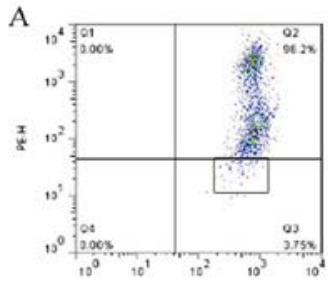

PBS + DMSO

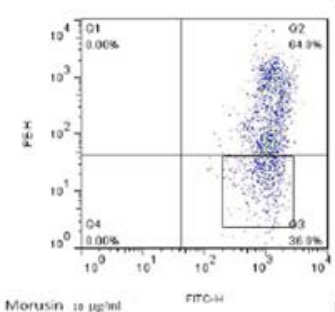

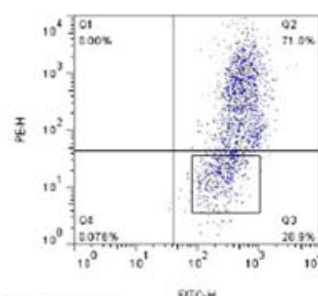

Morusin : $\mathrm{kit}$

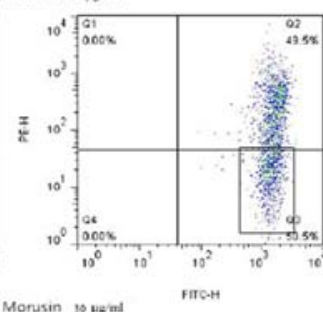

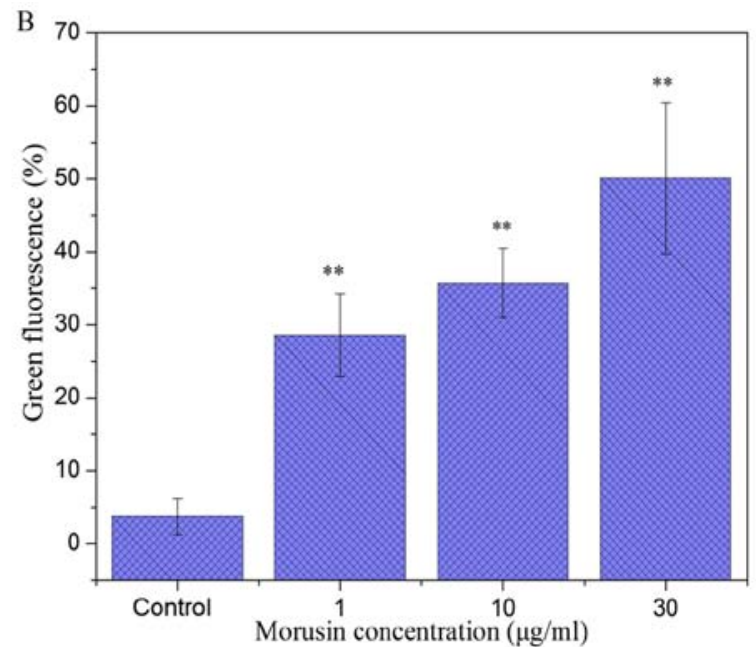

Figure 4. (A) Morusin-induced changes in the mitochondrial membrane potential $(\Delta \psi)$ in A549 cells, as assessed by JC-1 staining. (B) Quantification of cells with low mitochondrial transmembrane potentials. ${ }^{* *} \mathrm{P}<0.01$ vs. the control group.
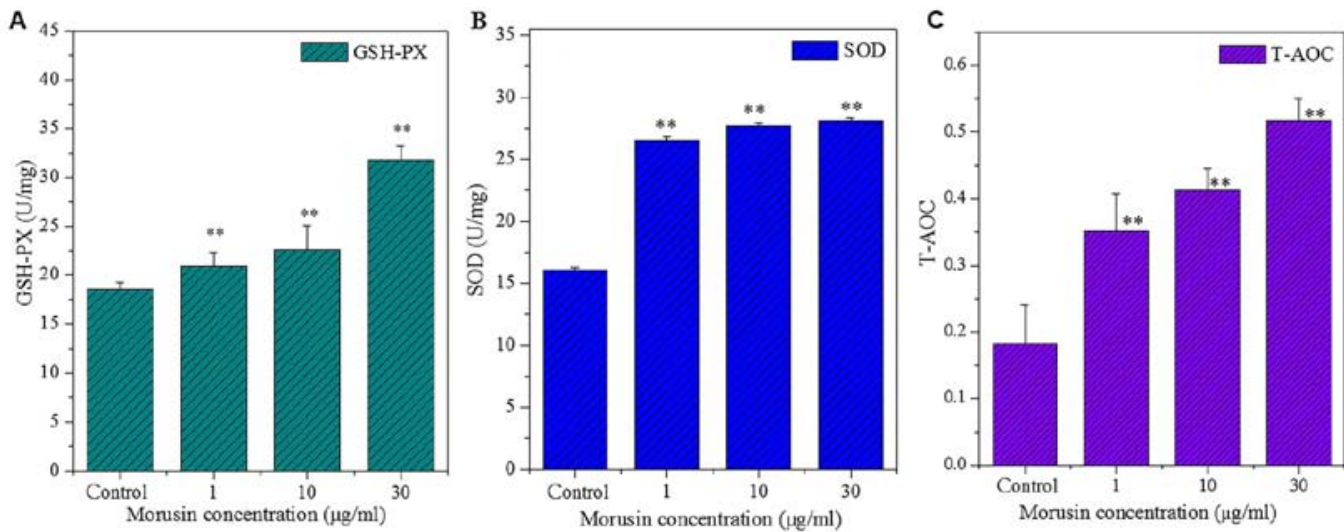

Figure 5. Morusin-induced increases in antioxidant activities. Morusin significantly increased the activity levels of (A) GSH-PX, (B) SOD and (C) T-AOC, in comparison with those of the control group $(\mathrm{P}<0.01)$, in a dose-dependent manner. ${ }^{* *} \mathrm{P}<0.01$ vs. the control group.
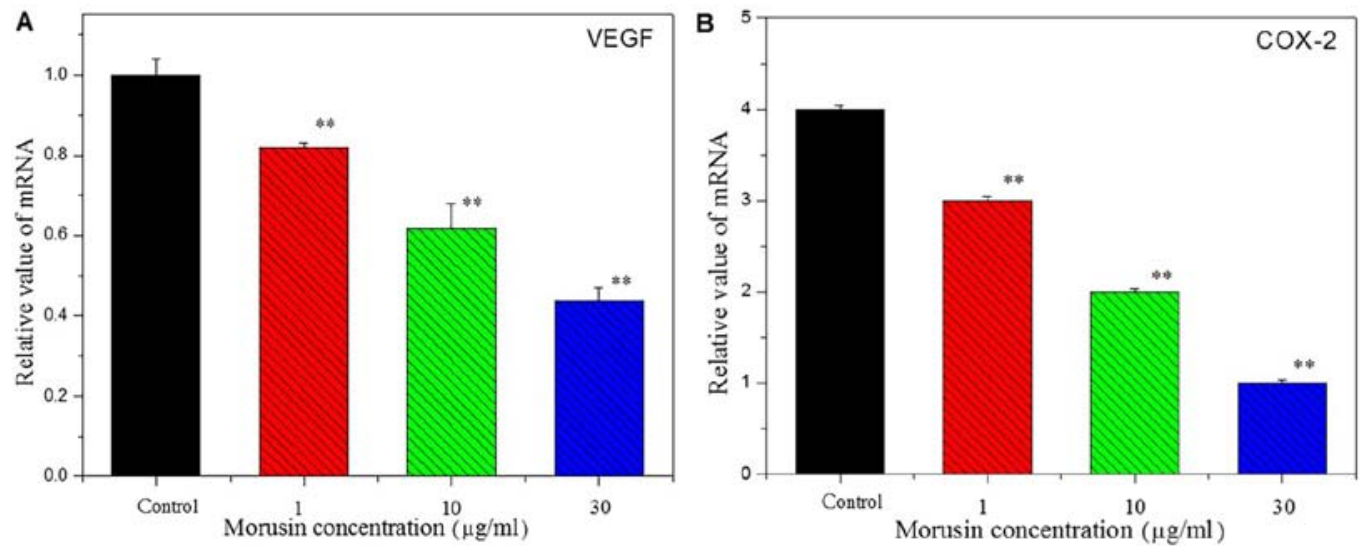

Figure 6. Relative expression of the (A) VEGF gene and the (B) COX-2 gene in A549 cells. ${ }^{* *} \mathrm{P}<0.01$ vs. the control group.

\section{Discussion}

The A549 human lung carcinoma cell line possesses a vigorously proliferative ability. The dysregulation and indefinite proliferation of cells is the foundation of the occurrence and development of tumors. Thus, analysis of the cell cycle in tumors is often used for anticancer drug screening and the evaluation of anticancer effects. Certain natural products play important roles in the development of drugs. For example, flavonoids exhibit a variety of biological activities that include anti-proliferative and anti-angiogenic activities. Many studies have demonstrated that morusin, an isoprenylated flavone 
from the mulberry tree, exerts inhibitory effects on cell proliferation. Therefore, morusin is regarded as a chemopreventive agent. The present study observed the cytotoxic effect of morusin on the A549 cell line in vitro.

The MTT assay is one of the most commonly used methods for assessing cell survival rates. The results of the present study indicated that morusin significantly inhibited the proliferation of A549 cells in a concentration-dependent manner. Within the $1-30 \mu \mathrm{g} / \mathrm{ml}$ concentration range, the cell survival rates gradually decreased with increasing concentrations and durations of morusin treatment. Since those concentrations affected cell viability, the effects observed in subsequent experiments may be due to the effects of cell viability. Chen et al found that the survival rate of SK-Hep1 cells was significantly reduced following treatment with $25-70 \mu \mathrm{M}$ morusin for $24 \mathrm{~h}$ (22). These experimental results were consistent with those of our study. Additionally, the morphological changes of the A549 cells were observed by inverted microscopy following the MTT assays. The results revealed that the numbers of adherent cells were significantly decreased in the morusin-treated groups in comparison with that of the control group. In addition, cell shrinkage and cracking were also observed in morusin-treated groups. Furthermore, Annexin V-FITC/PI double staining was used to detect cell apoptosis. The analyses indicated that the cells were primarily in apoptotic stage following morusin treatment. With increasing concentrations of morusin, the numbers of apoptotic cells significantly increased, a result that was consistent with the results of the MTT assay.

Mitochondria occupy a core position in cell survival and play a vital role in the cell apoptosis pathway. Mitochondrial morphology and functions are substantially altered when cells undergo apoptosis. The loss of $\Delta \psi$ can result in an increase in mitochondrial permeability and the release of cytochrome $c$. Cytochrome $c$ and apoptotic proteases are activating factors, and can combine with caspase- 9 to form a polymer complex that subsequently activates caspase-3. Activated caspase-3 induces the caspase-mediated apoptotic pathway, subsequently causing apoptosis $(32,33)$. In summary, morusin induces the loss of $\Delta \psi$ and causes cell apoptosis in human A549 lung carcinoma cells via the mitochondrial pathway.

Reactive oxygen species (ROS) are a series of substances produced in the process of cell aerobic metabolism and have a toxic effect on cells. The overproduction of ROS, or reductions in ROS scavenging abilities, can cause oxidative stress in cells. Oxidative stress can lead to increases in tissue lipid peroxidation, which then causes oxidative damage to the DNA and, eventually, abnormal protein expression. The results of the present study revealed that morusin increased the levels of SOD, GSH-PX and T-AOC. Furthermore, these activities were enhanced with increasing concentrations of morusin and were significantly increased in comparison with that of the control group $(\mathrm{P}<0.01)$. The results indicated that morusin increased the ROS scavenging abilities of the cells and reduced oxidative stress by increasing the activities of SOD and GSH-PX, as well as the level of T-AOC to achieve its antitumor effects.

The migration of tumor cells is a key part of tumor infiltration and migration. Inhibition of the migratory abilities of tumor cells can reduce the recurrence rate and prolong the survival of postoperative tumor patients. The results of the present study demonstrated that the wound-healing rates of the morusin-treated cells were much slower than those of the untreated cells, indicating a reduced migratory capacity.

Modern medical research has revealed that tumor angiogenesis is essential for tumor growth and migration (34). Tumor vasculature can provide sufficient nutrition for tumor cells. A series of angiogenic factors exist in the human body, among which COX-2 and VEGF are the most closely related to tumor angiogenesis (35). COX-2 is a type of induction enzyme that is only generated by stimulation from associated cytokines, tumor inducers and tumor genes. Cao et al have reported that COX-2 is a common feature of neoplasms, particularly those with an epithelial origin (36), and that COX-2 inhibitors exhibit good antitumor effects (37). VEGF is an important angiogenesis-promoting factor that can promote the growth and migration of tumor cells and is highly expressed during tumor angiogenesis (38) and in tumor cells (39). A number of Chinese herbal components have demonstrated that anti-angiogenic effects can be achieved by targeting VEGF-induced angiogenesis (40). In the present study, RT-PCR results indicated that COX-2 and VEGF genes in the morusin-treated A549 cells were significantly decreased in a concentration-dependent manner. Overall, these results indicated that morusin could inhibit tumor angiogenesis and affect the migration and invasion of tumor cells.

In conclusion, morusin significantly inhibited the proliferation of human A549 lung carcinoma cells and promoted cell apoptosis, as demonstrated by MTT assays and flow cytometry. In addition, morusin induced cell apoptosis via the loss of mitochondrial membrane potential. Furthermore, some antioxidant abilities were detected, indicating that morusin can enhance antioxidant capacity. Furthermore, the research further examined the effects of morusin on the genes that were associated with tumor angiogenesis. The results indicated that, following treatment with various concentrations of morusin, the expression levels of the COX-2 and VEGF genes in the A549 cells were significantly reduced in a dose-dependent manner. In general, morusin affected the invasion and migration of tumor cells via the inhibition of tumor angiogenesis. However, the specific mechanisms remain unclear and require further research.

\section{Acknowledgements}

Not applicable.

Funding

The present study was supported by the earmarked fund (CARS-18-ZJ0502) from the China Agriculture Research System and funding from the Priority Academic Program Development of Jiangsu Higher Education Institutions, P.R. China.

\section{Availability of data and materials}

The datasets used during the present study are available from the corresponding author upon reasonable request.

\section{Authors' contributions}

YQZ conceived this study. YL and SW performed most of the experiments and constructed the database. XLY performed some 
experiments, the statistical analysis, and wrote the manuscript. YQZ revised the manuscript. All authors read and approved the manuscript and agree to be accountable for all aspects of the research in ensuring that the accuracy or integrity of any part of the work are appropriately investigated and resolved.

\section{Ethics approval and consent to participate}

The present study was approved by the Ethics Committee of Soochow University.

\section{Consent for publication}

Not applicable.

\section{Competing interests}

The authors state that they have no competing interests.

\section{References}

1. Parkin DM, Bray F, Ferlay J and Pisani P: Estimating the world cancer burden: Globocan 2000. Int J Cancer 94: 153-156, 2001.

2. Beadsmoore CJ and Screaton NJ: Classification, staging and prognosis of lung cancer. Eur J Radiol 45: 8-17, 2003.

3. Parkin DM, Bray F, Ferlay J and Pisani P: Global cancer statistics, 2002. CA Cancer J Clin 55: 74, 2005.

4. Kim SY, Gao JJ, Lee WC, Ryu KS, Lee KR and Kim YC: Antioxidative flavonoids from the leaves of Morus alba. Arch Pharm Res 22: 81-85, 1999.

5. Asano N, Yamashita T, Yasuda K, Ikeda K, Kizu H, Kameda Y, Kato A, Nash RJ, Lee HS and Ryu KS: Polyhydroxylated alkaloids isolated from mulberry trees (Morus alba L.) and silkworms (Bombyx mori L). J Agric Food Chem 49: 4208-4213, 2001.

6. Oh H, Ko EK, Jun JY, Oh MH, Park SU, Kang KH, Lee HS and Kim YC: Hepatoprotective and free radical scavenging activities of prenylflavonoids, coumarin, and stilbene from Morus alba. Planta Med 68: 932-934, 2002.

7. Qiu F, Komatsu K, Kawasaki K, Saito K, Yao XS and Kano Y: A novel stilbene glucoside, oxyresveratrol 3'- $O$ - $\beta$-glucopyranoside, from the root bark of Morus alba. Planta Med 62: 559-561, 1996

8. Piao SJ, Qiu F, Chen LX, Pan Y and Dou DQ: New stilbene, benzofuran, and coumarin glycosides from Morus alba. Helvetica Chimica Acta 92: 579-587, 2009.

9. Piao SJ, Chen LX, Kang N and Qiu F: Simultaneous determination of five characteristic stilbene glycosides in root bark of Morus alba L. (Cortex mori) using high-performance liquid chromatography. Phytochem Anal 22: 230-235, 2011.

10. Nomura T, Fukai T, Yamada S and Katayanagi M: Studies on the constituents of the cultivated mulberry tree. I. Three new phenylflavones from the root bark of Morus alba L. Chem Pharm Bul 26: 1394-1402, 1978.

11. Ferlinahayati, Syah YM, Juliawaty LD, Achmad SA, Hakim EH, Takayama H, Said IM and Latip J: Phenolic constituents from the wood of Morus australis with cytotoxic activity. Z Naturforsch C 63: 35-39, 2008

12. Suhartati T, Yandri AS, Hadi S and Jhons FS: Morusin, a Bioactive Compound from the Root Bark of Artocarpus dadah. Eur J Sci Res 38: 643-648, 2009.

13. Mazimba O, Majinda RRT and Motlhanka D: Antioxidant and antibacterial constituents from Morus nigra. Afc J Pharm Pharmaco 5: 751-754, 2011

14. Guptaa G, Duaa K, Kazmi I and Anwar F: Anticonvulsant activity of Morusin isolated from Morus alba: Modulation of GABA receptor. Biomed Aging Pathology 4: 29-32, 2014.

15. Luo SD, Nemec J and Ning BM: Anti-HIV flavonoids from Morus alba. Acta Botanica Yunnanica 17: 89-95, 1995.

16. Cho JK, Ryu YB, Curtis-Long MJ, Kim JY, Kim D, Lee S, Lee WS and Park KH: Inhibition and structural reliability of prenylated flavones from the stem bark of Morus lhou on b-secretase (BACE-1). Bioorg Med Chem Lett 21: 2945-2948, 2011.

17. Kim JY, Lee WS, Kim YS, Curtis-Long MJ, Lee BW, Ryu YB and Park KH: Isolation of cholinesterase-inhibiting flavonoids from Morus lhou. J Agr Food Chem 59: 4589-4596, 2011.
18. Yoshizawa S, Suganuma M, Fujiki H, Fukai T, Nomura T and Sugimura T: Morusin, isolated from root bark of Morus alba L., inhibits tumour promotion of teleocidin. Phytotherapy Res 3: 193-195, 1989.

19. Fujiki H, Suganuma M, Takagi K, Yoshizawa S, Furuya HS, Yoshizawa S, Nishiwaki S, Kobayashi M, Okuda T, Nomura T, et al: Sarcophytols A and B, (-)-epigallocatechin gallate (EGCG), and morusin, anticarcinogenesis radiation Protection 2: 357-362, 1991.

20. Wang F, Zhang D, Mao J, Ke XX, Zhang R, Yin C, Gao N and Cui H: Morusin inhibits cell proliferation and tumor growth by down-regulating c-Myc in human gastric cancer. Oncotarget 8: 57187-57200, 2017.

21. Zhao JL: Morusin induces human colorectal cancer cell death via apoptosis, National Cheng Kung University, 2003.

22. Lee JC, Won SJ, Chao CL, Wu FL, Liu HS, Ling P, Lin CN and Su CL: Morusin induces apoptosis and suppresses NF- $\kappa$ B activity in human colorectal cancer HT-29 cells. Biochem Biophys Res Commun 372, 236-242, 2008.

23. Lin WL, Lai DY, Lee YJ, Chen NF and Tseng TH: Antitumor progression potential of morusin suppressing STAT3 and NFKB in human hepatoma SK-Hepl cells. Toxicol Lett 232: 490-498, 2015.

24. Kim C, Kim JH, Oh EY, Nam D, Lee SG, Lee J, Kim SH, Shim BS and Ahn KS: Blockage of STAT3 signaling pathway by morusin induces apoptosis and inhibits invasion in human pancreatic tumor cells. Pancreas 45: 409-419, 2016.

25. Wang L, Guo H, Yang L, Dong L, Lin C, Zhang J, Lin P and Wan X: Morusin inhibits human cervical cancer stem cell growth and migration through attenuation of $\mathrm{NF}-\kappa \mathrm{B}$ activity and apoptosis induction. Mol Cell Biochem 379: 7-18, 2013.

26. Wan LZ, Ma B and Zhang YQ: Preparation of morusin from Ramulus mori and its effects on mice with transplanted H22 hepatocarcinoma. Biofactors 40: 636-645, 2014.

27. Ding B, Lv Y and Zhang YQ: Anti-tumor effect of morusin from the branch bark of cultivated mulberry in Bel-7402 cells via the MAPK pathway. Rsc Adv 6: 17396-17404, 2016.

28. Mosmann T: Rapid colorimetric assay for cellular growth and survival: Application to proliferation and cytotoxicity assays. J Immunol Medthods 65: 55-63, 1983.

29. Petit PX, Susin SA, Zamzami N, Mignotte B and Kroemer G: Mitochondria and programmed cell death: Back to the future. FEBS Lett 396: 7-13, 1996.

30. Prager GW, Lackner EM, Krauth MT, Unseld M, Poettler M, Laffer S, Cerny-Reiterer S, Lamm W, Kornek GV, Binder BR, et al: Targeting of VEGF-dependent transendothelial migration of cancer cells by bevacizumab. Mol Oncol 4: 150-160, 2010.

31. Gong $\mathrm{C}, \mathrm{Xu} \mathrm{C}, \mathrm{Ji} \mathrm{L}$ and Wang $\mathrm{Z}$ : A novel semi-synthetic andrographolide analogue A5 inhibits tumor angiogenesis via blocking the VEGFR2-p38/ERK1/2 signal pathway. Biosci Trends 7: 230-236, 2013.

32. Chen LH, Fang J, Li H, Demark-Wahnefried W and Lin X: Enterolactone induces apoptosis in human prostate carcinoma LNCaP cells via a mitochondrial-mediated, caspase-dependent pathway. Mol Cancer Ther 6: 2581-2590, 2007.

33. Chen M, Guerrero AD, Huang L, Shabier Z, Pan M, Tan TH and Wang J: Caspase-9-induced mitochondrial disruption through cleavage of anti-apoptotic BCL-2 family members. J Biol Chem 282: 33888-33895, 2007.

34. Sikora J: Tumor angiogenesis in human lung adenocarcinoma. Cancer 76: 915-916, 1995

35. Kim TJ, Lee YS, Kang JH, Kim YS and Kang CS: Prognostic significance of expression of VEGF and Cox-2 in nasopharyngeal carcinoma and its association with expression of C-erbB2 and EGFR. J Surg Oncol 103: 46-52, 2011.

36. Rundhaug JE, Mikulec C, Pavone A and Fischer SM: A role for cyclooxygenase-2 in ultraviolet light-induced skin carcinogenesis. Mol Carcinog 46: 692-698, 2007.

37. Cao Y and Prescott SM: Many actions of cyclooxygenase- 2 in cellular dynamics and in cancer. J Cell Physiol 190: 279-286, 2002.

38. Bremnes RM, Camps C and Sirera R: Angiogenesis in non-small cell lung cancer: The prognostic impact of neoangiogenesis and the cytokines VEGF and bFGF in tumours and blood. Lung Cancer 51: 143-158, 2006.

39. Ferrara N and Kerbel RS: Angiogenesis as a therapeutic target. Nature 438: 967-974, 2005.

40. Izuta H, Shimazawa M, Tsuruma K, Araki Y, Mishima S and Hara $\mathrm{H}$ : Bee products prevent VEGF-induced angiogenesis in human umbilical vein endothelial cells. BMC Complement Altern Med 9: 45, 2009. 